\title{
Predicting Mother and Child Emotional Availability in Singaporean Bilingual English and Mandarin Dyads: A Multilevel Approach to the Specificity Principle
}

\author{
Gianluca Esposito ${ }^{1,2,3,}$, Bindiya L. Raghunath ${ }^{1}$, Atiqah Azhari ${ }^{1}$, Peipei Setoh ${ }^{1,{ }^{*}}$, and \\ Marc H. Bornstein ${ }^{4,5,6}$
}

\footnotetext{
${ }_{2}^{1}$ Psychology Program, School of Social Sciences, Nanyang Technological University, Singapore

${ }^{2}$ Lee Kong Chian School of Medicine, Nanyang Technological University, Singapore, Singapore

${ }^{3}$ Department of Psychology and Cognitive Science, University of Trento, Rovereto, TN, Italy MD, USA

${ }^{5}$ UNICEF, New York City, NY, USA

${ }^{6}$ Institutes for Fiscal Studies, London, United Kingdom

*Corresponding author/s: gianluca.esposito@ntu.edu.sg and/or gianluca.esposito@unitn.it
}

${ }^{4}$ Eunice Kennedy Shriver National Institute of Child Health and Human Development National Institutes of Health, Bethesda,

\begin{abstract}
When interacting with one another, bilingual caregivers and their young bilingual children can switch from one language to another to convey emotion and information in meaningful ways. The Specificity Principle (Bornstein, 2017) states that specific setting conditions affect specific outcomes in specific ways in specific individuals at specific times. Here we tested three constituents of the Specificity Principle: We evaluated how the language used in interaction (setting condition) differentially affects different dimensions of emotional availability (outcomes) in mothers and children (individuals). Twenty-six Singaporean English-Mandarin bilingual mother-child dyads (Mother $M$ age $=33$ years; Child $M$ age $=19$ months) participated in two counterbalanced play sessions, one exclusively in English and one exclusively in Mandarin. Using recursive-partitioning analyses, we assessed (i) how child language dominance, dimensions of maternal emotional availability, and mother-child physiological synchrony accounted for dimensions of child emotional availability and (ii) how child language dominance, child emotional availability, and mother-child physiological synchrony accounted for overall maternal emotional availability. In agreement with predictions from the Specificity Principle, our results show that different predictors of different dimensions of child and mother emotional availability differ according to whether the mother interacted with her child in the child's dominant or nondominant language. The findings suggest that language specificity influences the quality of mother-child interactions. Limitations and future research directions are discussed.
\end{abstract}

Keywords: Mother-child Emotional Availability; HRV Synchrony; Bilingual Dyad Interaction; Specificity Principle

Acknowledgements. This research was supported by the Nanyang Technological University NAP SUG Grant (GE), Singapore Ministry of Education's Academic Research Fund Tier 1 (GE;PS), Social Science Research Thematic Grant (MOE2016SSRTG-017, PS), Intramural Research Program of the NIH/NICHD, USA, (MHB) and an International Research Fellowship at the Institute for Fiscal Studies (IFS), London, UK, funded by the European Research Council (ERC) under the Horizon 2020 research and innovation programme (grant agreement No 695300-HKADeC-ERC-2015-AdG, MHB).

Notes on contributors: Conceptualization, GE, MHB, and PS; analysis, BLR; investigation, GE, BLR, AA, and PS; data curation, LBR; writing-original draft preparation, BLR and MHB; writing-review and editing, GE, MHB; visualization, BLR and $A B$; funding acquisition GE, MHB, and PS. 


\section{Emotional Availability and Physiological Synchrony in Mother-Child Interactions}

The quality of mother-child interaction plays a crucial role in child development, especially in the early years as children learn to experience and interpret the world based on interactions with their caregivers (Bornstein, 2015; Fuertes et al., 2017; Venuti et al., 2009). Emotional availability (EA; Emde, 1980) is a dyadic construct that reflects the quality of emotional exchanges between partners and underpins effective mother-child interactions (Biringen, 2000; Biringen \& Robinson, 1991; Bornstein et al., 2012; Saunders et al., 2015). The Emotional Availability Scales (EAS; Biringen, 2008) is a global measure of dyadic interaction that operationalizes EA into four parental (sensitivity, structuring, non-intrusiveness, and non-hostility) and two child domains (responsiveness and involvement of the parent) which, together, constitute telling indicators of dyadic functioning (Biringen \& Robinson, 1991; Bornstein et al., 2012; Emde, 2000; Emde \& Easterbrooks, 1985; Saunders et al., 2015; Venuti et al., 2009). Under the EA framework, mother and child bidirectionally influence one another and dynamically change as a result, so as to mutually shape the quality of their interaction (Sameroff, 2009, 2010). Supporting this theory, Stack and colleagues (2012) demonstrated that optimal levels of (i) maternal structuring, where mothers provide their child with appropriate scaffolding and a supportive framework for interaction, as well as (ii) maternal sensitivity, a characteristic of mothers who respond to their child in a warm and flexible manner, predict greater child responsiveness. The child's emotional availability likewise affects maternal behaviors. For instance, more responsive children elicit greater maternal sensitivity (Engfer \& Gavranidou, 1987; TamisLeMonda et al., 2009). These findings attest to the mutual interaction between mother and child emotional availability in the context of dyadic interaction.

Mother-child interactions that display greater emotional availability typically emerge when partners respond to each other positively, constructively, and synchronously (Longhi, 2009; Thompson \& Trevathan, 2009). Behavioral synchrony is the temporal coordination of action events between mother and child, such as matched facial expressions and gazes, that suggest the sensitivity of each partner to the other's emotional state (Bornstein, 2013a; Leclère et al., 2014). Synchrony is manifested behaviorally and is supported by the entrainment of maternal and child physiological systems (Feldman, 2007); enhanced interactional synchrony is associated with concordance in mother and child physiological arousal (Feldman et al., 2010). The burgeoning literature on physiological synchrony has revealed that timedomain parameters of the autonomic nervous system, such as inter-beat interval (IBI; the temporal distance between consecutive heart beats), capture nuanced changes in mother-child physiological synchrony (Creaven et al., 2014). In support of this point, IBI synchrony in preschool-aged children and their mothers is increased during dyadic interaction (Suveg et al., 2015). Likewise, physiological synchrony in school-aged children and their mothers is greater during positive interactions that are preceded by conflictual interactions, suggesting that physiological synchrony might be essential in reparative interactive processes (Woltering et al., 2015). Reciprocally, physiological synchrony between partners during non-optimal conflict situations predicts negative emotions and physiological inflammation (Wilson et al., 2018). These studies document the importance of considering specific qualities of emotional engagement when assessing and interpreting physiological synchrony in mother-child interactions.

\section{Language Choice and Dominance in Bilingual Dyads}

The language which mothers choose to use to interact with their children is a matter of significance in bilingual mother-child dyads. Germane to the present study, a parent's choice of language influences the emotional climate of parent-child interactions. Bilingual parents experience each of their languages differently, and they convey different emotions to their children through their specific language choices (see Chen et al., 2012). Parents' language choice when interacting with their children affords profound emotional meaning and influences the degree of connection in parent-child relationships (De Houwer, 2009; Portes \& Rumbaut, 2001). Appreciating the significance of parent language 
choice has penetrated outside traditional academic venues where parenting guides propose that interacting with a child in their native language promotes emotional intimacy in the dyad (Baker, 2014; Tokuhama-Espinosa, 2003).

Thus, a bilingual child's proficiency in the language that his/her parent chooses for interaction can substantially influence the quality of their interaction. Bilingual children typically receive unequal exposure to their two languages and use each of their languages to different extents, which leads to dominance in one language over the other (Bosch \& SebastiánGallés, 2001; De Houwer, 2015; De Houwer \& Bornstein, 2016; Grüter \& Paradis, 2014). Extant cognitive and physiological studies indicate that bilinguals experience greater emotional arousal when, for example, conversing about past memories, viewing movies, and processing emotionally laden words in their dominant language (Pavlenko, 2012). Speaking in a dominant compared to a non-dominant language even impacts older children's emotional experiences when interacting with their parents. Bilingual immigrant youth experience less emotional engagement conversing with their parents when they use their dominant non-native language, but have parents who respond in their weaker native language (Hua, 2008). Similarly, respondents to an online questionnaire indicated that verbal expressions of intimacy (such as "I love you") bear greater emotional weight when uttered in the respondent's dominant language compared to a non-dominant language (Dewaele, 2008). These studies point to the centrality of language dominance in influencing social and emotional experiences in bilingual speakers (Bornstein, 2013b).

Because language dominance exerts distinct socioemotional effects in bilingual individuals, a mother's choice of language when interacting with her child may elicit different patterns of mother-child interaction, depending on whether the child's dominant or non-dominant language is in play. To date, the literature has identified the importance of emotional availability in motherchild interactions without accounting for a possible pivotal role of language dominance or its implications in bilingual dyads. To investigate how a child's language dominance moderates emotional availability in mother-child interactions, we drew on the Specificity Principle. The Specificity Principle asserts that specific setting conditions affect specific outcomes of specific individuals in specific ways at specific times (Bornstein, 2017; Bornstein et al., 2017). In accordance with this principle, the dominant visà-vis non-dominant language (setting condition) in which interaction occurs could differentially affect the relative importance of different dimensions of emotional availability (outcomes) in mothers and children (individuals).

\section{Bilingualism in Singapore}

Singapore instituted a bilingual language policy in 1966, where all Singaporeans advancing through the formal education system from Age 7 to at least Age 16 years learn both English and their choice of a "mother tongue" language, which is typically Mandarin for Chinese Singaporeans, Malay for Malay Singaporeans, and Tamil for Indian Singaporeans. Hence bilingualism is essentially universal and the default in Singapore, so young Singaporeans are either simultaneous or sequential bilingual language learners. As a citystate that actively supports the dual acquisition of English as the official lingua franca of instruction alongside a native "mother tongue" from a young age, bilingual Singaporean dyads afford a unique opportunity to examine the effects of language dominance in a society where bilingualism is the societal norm.

Relevant to the present study, bilingual English-Mandarin Singaporean mothers express their thoughts more in English but tend to convey desires through their native Mandarin (Cheng et al., 2020). The specific usage of each language for distinct functions could differentially shape the course of mother-child interaction, depending on which language the mother is using (Setoh et al., in press). Any associations between the use of a child's dominant compared to non-dominant language and the quality of mother-child interaction in English-Mandarin bilingual dyads is an open question.

\section{The Present Study}

This study aimed to investigate specificities of mother-child emotional availability based on 
mothers' use of their child's dominant and nondominant language. We evaluated relative influences of maternal EA on child EA, and child $E A$ on maternal $E A$, along with mother-child physiological synchrony, in English-Mandarin Singaporean bilingual dyads during interactions in which dyads spoke exclusively English or Mandarin. Using a data driven approach, the main questions we investigated were: (1) how child language dominance, dimensions of maternal emotional availability, and mother-child physiological synchrony account for dimensions of child emotional availability and (2) how child language dominance, child emotional availability, and mother-child physiological synchrony account for overall mother emotional availability across two linguistic contexts. We embarked on this study with two main hypotheses. Employing the Specificity Principle, we expected different hierarchical patterns among the same predictors in the child language dominant versus nondominant sessions. Specifically, we expected that the child's language dominance would be more important in predicting the child's emotional availability than the other predictor variables (dimensions of maternal emotional availability and mother-child physiological synchrony) in the child's dominant language session, but not in the non-dominant session (Hypothesis1). We also expected a similar pattern of results in predicting overall maternal emotional availability: Child language dominance would be more important in predicting maternal emotional availability than the other predictor variables (dimensions of child emotional availability and mother-child physiological synchrony) in the child's dominant language session, but not in the non-dominant session (Hypothesis2).

\section{Methods}

\section{Sample}

Twenty-six typically developing bilingual English-Mandarin Singaporean mother-child dyads took part in the study, including 14 female and 12 male children, aged between 16 and 24 months $(M=19.38$ months, $S D=2.08)$. Of the 26 mothers $(M=33.12$ years, $S D=2.88), 22$ were employed, two were currently unemployed, and two were homemakers. During recruitment, only mothers who confirmed that they used both languages frequently were eligible to take part in the study. Mothers were only required to have basic conversations in both their and their child's languages (English and Mandarin). All mothers held either a diploma or a university degree qualification and reported an above-average to high monthly household income ranging between SG $\$ 5,000$ and SG\$10,000 (Singapore. Department of Statistics Singapore, 2019). Mothers provided written informed consent, and the study was approved by the Institutional Review Board of Nanyang Technological University, Singapore (Protocol number: 201411-010-07).

\section{Design}

Two play sessions took place on the same day at the dyad's home. A standard set of ageappropriate toys (Bornstein et al., 1996) was set on a carpet for both play sessions. Toys included a foam rubber ball, train, doll, small blanket, tea set, cell phone, stacking cups, and two small identical picture books (one in English, one in Mandarin). The toys represented gender-neutral, masculine, and feminine categories (see Caldera et al., 1989) that allowed for play that varied from simple exploration to relatively complex symbolic play (Bornstein, 2007). Mothers were instructed to play with their child as they normally would, only use the toys provided, and remain on the carpet with their child during the play sessions. The two 10-min play sessions were counterbalanced, one where the mother spoke exclusively English and one where the mother spoke exclusively Mandarin. To collect ECG data during the two play sessions the mother and child each wore a Comftech Electrocardiography (ECG) belt slightly above the waist. Both play sessions were videorecorded for offline behavioral coding.

\section{Child Language Dominance}

Short-form versions of MacArthur-Bates Communicative Development Inventory (MCDI; English version: Fenson et al., 2007; and Mandarin version: Tardif \& Fletcher, 2008) vocabulary checklists for American English and Mandarin were used to determine each child's productive language and dominance. Mothers checked all English and Mandarin words that their child produced verbally. The numbers of nouns and verbs as well as the distribution of nouns and verbs varied slightly between the two 
vocabulary lists. The English version had a total of 100 words (50 nouns, 19 verbs, and 31 other words such as pronouns, quantifiers, etc.), and the Mandarin version had a total of 113 words (48 nouns, 29 verbs, and 36 other words). Therefore, the percentages of words each child produced verbally for English and Mandarin were calculated. A child's language dominance score (LDS) was then calculated as the percentage of English to Mandarin, such that a greater positive LDS score indicated dominance in English whereas a greater negative score indicated dominance in Mandarin.

\section{Mother and Child Emotional Availability}

Mother and child emotional availability were evaluated from videorecords by two trained, reliable, independent coders using the Emotional Availability Scales: Infancy to Early Childhood Version (EAS 4th ed.; Biringen, 2008). There are four dimensions of maternal emotional availability, and two dimensions of child emotional availability. Maternal sensitivity assesses a mother's acceptance and flexibility towards her child, and maternal structuring reflects the extent to which mothers provide appropriate scaffolding and organization of her child's activities by providing rules, regulations, and a supportive framework for the interaction without compromising the child's interest in such activities. Maternal non-intrusiveness refers to maternal support for the child without interrupting the child by being over-directive, overstimulating, overprotecting, or interfering, and maternal nonhostility assesses whether mothers behave in a way that is patient and harmonious and not rejecting, abrasive, or antagonistic. The two child dimensions of EAS, child responsiveness and child involvement, evaluate age- and contextappropriate exploring and reacting to the mother's bids and the child's overall enjoyment of the interaction and the child's ability, willingness, and success in engaging the mother in interaction, respectively. Scores for each dimension of emotional availability could range from 1 (non-optimal) to 7 (optimal). Before the experiment, mothers were instructed to prompt their child to speak in the language of the condition if her child ever spoke in the other language; maternal sensitivity was coded based on the mother's interaction with the child interaction independent of the language. Coder reliability (kappa; Cohen, 1960, 1968 ) on $20 \%$ of the video records coded by two coders independently was .94 for EA in English play sessions (Maternal sensitivity: 0.94, maternal structuring: 0.78 , maternal nonintrusiveness: 0.94 , maternal non-hostility: 1.00 , child responsiveness: 1.00 , child involvement: 0.95 ) and 0.93 for EA in Mandarin play sessions (Maternal sensitivity: 0.80 , maternal structuring: 1.00, maternal non-intrusiveness: 0.76 , maternal non-hostility: 1.00 , child responsiveness: 1.00 , child involvement: 1.00)

\section{Mother and Child Physiological Synchrony}

Offline signal processing of ECG data consisted of high-pass and low-pass signal filtering, followed by the determination of R-R peaks. The distance between two consecutive $R$ $R$ peaks is called the inter-beat interval (IBI). After extracting $\mid \mathrm{BI}$ values, a time-domain index of heart rate variability, rMSSD, was calculated. rMSSD is the square root of the mean of the sums of squares of differences between adjacent IBIs calculated over a moving 10-s window with 5-s steps. To obtain mother-child physiological synchrony, maximum cross-correlation calculation was applied to assess the commonalities between the mother's and child's physiological time course, the rMSSD index, while mother and child were involved in the same play activity. Cross-correlation analysis indicates the extent to which physiological time courses covary, while taking into account any lags in responses. Mother-child physiological synchrony was computed through maximum crosscorrelation of the mother's and child's rMSSD indexes with a lag of $\pm 5 \mathrm{~s}$ (Golland et al., 2014), using Pysynch library (Bizzego et al., 2019). A greater correlation index represented greater physiological synchrony between mother and child.

\section{Analytical Plan}

In preliminary analyses, correlations among child LDS, mother and child EA, and mother-child physiological synchrony for the English and Mandarin play sessions were assessed. To reduce the number of dependent variables to be predicted in our models, an overall measure of maternal EA was computed as the average of the four maternal scales: sensitivity, structuring, non-intrusiveness, and non-hostility. However, 
the four maternal EA scales were treated separately when used as predictors. Pairedsample t-tests were then conducted to evaluate differences in mother and child emotional availability and physiological synchrony during English compared to Mandarin play sessions.

Given the relatively small sample size and limitations of statistical power, we employed a novel analytic path, exploratory recursive partitioning (regression tree-based models), to investigate (1) how child LDS, maternal EA, and mother-child physiological synchrony account for dimensions of child EA and (2) how child LDS, child EA, and mother-child physiological synchrony account for maternal EA during English-language play and Mandarin-language play separately. Recursive partitioning explores data relations with easy-to-visualize decision rules to predict a continuous outcome ${ }^{1}$ (Costello et al., 2003; Esposito et al., 2017).

Recursive partitioning is achieved in two steps: first, in the growing step, the model explores all possible relations among variables and, second, in the pruning step, values of the independent variables stratify the dependent variable into two subsamples that differ statistically. Tree-based models uncover the hierarchical importance of independent variables that explain variance in the dependent variable along with the value of the independent variable that significantly stratifies the dependent variable into two parts, called low and high leaves. Recursive partitioning was calculated using the rpart package of statistical software R (ver. 4.113) with the minimum number of observations in any terminal node set to 4 . The tree allowed for maximum growth by setting the complexity parameter close to zero at 0.0002 and was subsequently pruned to obtain an optimal tree by

\footnotetext{
${ }^{1}$ We computed two regression analyses, one predicting child emotional availability from child language dominance, maternal emotional availability, and mother-child physiological synchrony, and a second predicting maternal emotional availability from child language dominance, child emotionality, and mother-child physiological synchrony. These analyses were not significant, presumably because of power limitations.
}

removing branches containing nodes with tvalues greater than $1.64(\alpha=.05)$.

\section{Results}

\section{Preliminary Analyses}

Correlations $(n=28$ for each language condition) between child LDS, mother EA (sensitivity, structuring, non-intrusiveness, and non-hostility), child EA (responsiveness and involvement), and mother-child physiological synchrony were assessed separately for the English-language and Mandarin-language play sessions. Correlation p-values used Bonferroni correction. Only one significant correlation emerged: Child LDS was positively correlated with maternal structuring during the Englishlanguage play session, $r(24)=.62, p<.05$, suggesting that greater child dominance in English is associated with greater maternal structuring during English-language play. Correlations between child and maternal EA during English-language and Mandarin-language play are reported in Table 1. During the Englishlanguage play session, the greater the involvement of the child, the better maternal structuring. During the Mandarin-language play session, the greater the responsiveness and involvement of the child, the better maternal sensitivity and structuring.

Paired-sample t-tests evaluated differences in LDS, EA, and physiological synchrony between the English-language and Mandarin-language play sessions. Overall, children were more dominant in English ( $\mathrm{M}$ percentage of English = 25.74, SD $=18.85)$ compared to Mandarin $(\mathrm{M}$ percentage of Mandarin $=9.43, \mathrm{SD}=13.38$ ), $\mathrm{t}(25)=-5.23, \mathrm{p}<.001, \mathrm{~d}=-1.03$. Only three children were more dominant in Mandarin. Thus, the majority of the children were dominant in English: Mean LDS (percentage of English percentage of Mandarin) in the sample is 16.30 $(\mathrm{SD}=15.89)$

\section{Exploratory Recursive Partitioning in Predicting Child Emotional Availability Child Responsiveness}

As shown in Figures $1 \mathrm{~A}$ and $\mathrm{B}$, the prediction of child responsive emotional availability followed different paths during English versus Mandarin play. 
English-Language Play. Figure $1 \mathrm{~A}$ shows that the path to greater child responsiveness during English-language play led from higher child English language dominance through higher maternal structuring to lower mother-child physiological synchrony when maternal structuring was low. Thus, child language dominance first partitioned child responsiveness at the LDS value of 1.57: Children with lower dominance in English $(<1.57$, i.e. the 'yes' branch of the Child Language Dominance $<1.57$ leaf) were the least responsive (4.83) during play. Among children who showed greater dominance in English ( $>1.57$, i.e. the 'no' branch of the Child Language Dominance $<1.57$ leaf), maternal structuring further partitioned child responsiveness at a value of 6.50 ; mothers who structured play more $(>6.50$, i.e. the 'no' branch of the Maternal Structuring $<6.50$ leaf) had more responsive children (6.25). When maternal structuring was lower $(<6.5$, i.e. the 'yes' branch of the Maternal Structuring $<6.50$ leaf), motherchild physiological synchrony further partitioned child responsiveness at an HRV Synchrony value of 0.21 : child responsiveness during play was higher (5.81) when there was lower mother-child physiological synchrony $(\leq 0.21$, i.e. the ' $n o$ ' branch of the HRV Synchrony $\geq 0.213$ leaf) as compared to when there was higher physiological synchrony ( $\geq 0.21$, i.e. the 'yes' branch of the HRV Synchrony $\geq 0.213$ leaf) during which the child responsiveness was lower (5.20).

Mandarin-Language Play. Figure 1B shows a different path to greater child responsiveness during Mandarin-language play: It led from maternal sensitivity to higher child Mandarin dominance. Maternal sensitivity partitioned child responsiveness twice in the tree model. First, at a value of 6.50: Children were more responsive (6.56) when their mothers displayed greater sensitivity ( $>6.5$, i.e. the 'no' branch of the Maternal Sensitivity < 6.50 leaf) during play. Second at a value of 5.50, when mothers displayed lower sensitivity $(<5.5$, i.e. the 'yes' branch of the Maternal Sensitivity < 5.50 leaf), children were least responsive during play (5.2). When maternal sensitivity fell between $5.5-6.5$, child language dominance further partitioned child responsiveness at the LDS value of 18.58: children who were more dominant in Mandarin
(LDS $\leq 18.58$, i.e. the 'no' branch of the Child Language Dominance $\geq 18.58$ leaf) were more responsive (6.0) during play compared to children who were less dominant in Mandarin (LDS $\geq 18.58$, i.e. the 'yes' branch of the Child Language Dominance $\geq 18.58$ leaf), during which child responsiveness was lower (5.5).

\section{Child involvement}

As shown in Figures $1 C$ and $D$, the prediction of child involvement emotional availability also followed different paths during English versus Mandarin play.

English-Language Play. Figure 1C shows that the path to greater child involvement during English-language play led from more maternal structuring through lower maternal intrusiveness. Maternal structuring first partitioned child involvement at a value of 5.50: Children involved their mothers less (4.88) during play when mothers structured play less $(<5.50$, i.e. the 'yes' branch of the Maternal Structuring $<5.50$ leaf). When maternal structuring was high $(>5.50$, i.e. the 'no' branch of the Maternal Structuring $<5.50$ leaf), maternal non-intrusiveness further partitioned child involvement at a value of 6.50 , such that children involved their mothers more (6.25) when their mothers structured the play more, and as partitioned next, when mothers were less intrusive (non-intrusiveness $>6.50$, i.e. the 'no ' branch of the Maternal Nonintrusiveness $<6.50$ leaf) during play.

Mandarin-Language Play. Figure 1D shows a different path to greater child involvement during Mandarin-language play; it led from higher maternal sensitivity to higher dominance in Mandarin, only when maternal sensitivity was low and maternal non-intrusiveness was high. Thus, maternal structuring first partitioned child involvement at a value of 6.50: Children involved their mothers more (6.67) when their mothers displayed greater sensitivity ( $>6.50$, i.e. the 'no' branch of the Maternal Sensitivity $<6.50$ leaf) during play. When maternal sensitivity was low $(<6.50$, i.e., the 'yes' branch of the Maternal Sensitivity < 6.50 leaf), maternal nonintrusiveness further partitioned child involvement at a value of 5.50: Among mothers who displayed low sensitivity, mothers who were more intrusive (non-intrusiveness $<5.50$, i.e. the 
'yes' branch of the Maternal Non-intrusiveness < 5.50 leaf) had children who involved them least (5.00) during play. However, with mothers who were less intrusive (non-intrusiveness $>5.50$, i.e. the 'no' branch of the Maternal Non-intrusiveness $<5.50$ leaf), child language dominance further partitioned child involvement at the LDS value of 18.58: children who were more dominant in Mandarin (LDS $\leq 18.58$, i.e., the ' $n o$ ' branch of the Child Language Dominance $\geq 18.58$ leaf) involved their mothers more (6.00) during play.

\section{Exploratory Recursive Partitioning in Predicting Maternal Emotional Availability}

As was true for two dimensions of child emotional availability, the prediction of overall maternal emotional availability followed different paths during English versus Mandarin play.

English-Language Play. Figure 2A shows that the path to greater overall maternal emotional availability during English-language play led from child English language dominance through greater child responsiveness. Child language dominance first partitioned overall maternal emotional availability at the LDS value of 1.57 : Overall maternal emotional availability was lower (5.38) when children were less dominant in English ( $<1.57$, i.e. the 'yes' branch of the Child Language Dominance < 1.57 leaf). When children were more dominant in English (> 1.57, i.e. the 'yes' branch of the Child Language Dominance < 1.57 leaf), child responsiveness further partitioned overall maternal emotional availability at a value of 5.50: Maternal emotional availability was greater (6.11) among children who were more responsive ( $>5.5$, i.e. the 'no' branch of the Child Responsiveness $<5.50$ leaf).

Mandarin-Language Play. Figure 2B shows that the path to greater overall maternal emotional availability during Mandarin-language play led from child involvement to lower child Mandarin language dominance. Child involvement first partitioned overall maternal emotional availability at a value of 5.50: Overall maternal emotional availability was lower (5.68) towards children who involved their mothers less during play $(<5.5$, i.e. the 'yes ' branch of the Child Involvement $<5.50$ leaf) their mothers. When children involved their mothers more during play ( $>5.50$, i.e. the ' $n o$ ' branch of the
Child Involvement < 5.50 leaf), child language dominance further partitioned overall maternal emotional availability at the LDS value of 6.79 : Maternal emotional availability was higher (6.46) when children involved their mothers more but were less dominant in Mandarin (> 6.79, i.e. the ' $n o$ ' branch of the Child Language Dominance < 6.75 leaf) and maternal emotional availability was less (6.08) to children who were more dominant in Mandarin ( $<6.79$, i.e. the 'yes' branch of the Child Language Dominance $<6.75$ leaf).

\section{Discussion}

Overall, taking into account limitations of the study (discussed in detail in the Limitations section), our results support to the Specificity Principle, which postulates (in part) that specific outcomes in specific individuals are differently affected under different specific conditions (Bornstein, 2017; Bornstein et al., 2017). Indeed, this experiment demonstrated how the same independent variables (child language dominance, either mother or child emotional availability, and mother-child physiological synchrony) differentially predict the same outcomes (child or mother emotional availability) in the same mother-child dyads in two contrasting conditions (English or Mandarin language play).

\section{Child Emotional Availability}

We first analyzed relations among child language dominance, maternal emotional availability, and mother-child physiological synchrony in predicting two facets of child emotional availability. As we expected, relations among the three independent variables differed in the child language-dominant English-language play session versus the child languagenondominant Mandarin-language play session and for the two facets of child emotional availability. During English-language motherchild interactions, child dominance in English (relative to Mandarin) along with greater maternal emotional availability, particularly maternal structuring, and lesser mother-child physiological synchrony (only when maternal structuring was low) predicted greater child responsiveness. Only maternal emotional availability, specifically maternal structuring and non-intrusiveness predicted greater child involvement. During 
Mandarin-language mother-child interactions, children's greater dominance in Mandarin and mothers' greater emotional availability predicted children's greater emotional availability (both responsiveness and involvement). Maternal sensitivity and maternal non-intrusiveness, respectively, contributed to child responsiveness and involvement.

In accord with our first hypothesis, during the English-language play session, which was the dominant language play session of the majority children (shown by the mean LDS of 16.30 of the sample), child language dominance in English was more predictive than maternal emotional availability for child responsiveness (but not for child involvement). A highly responsive child interacts with his or her mother when she reaches out and clearly enjoys doing so. A highly involved child invites his or her mother to join the child in play (Saunders et al., 2015). Considering the very young age of our child participants, it might be that language dominance was more impactful in responding to maternal prompts, but the differences were not so evident in terms of child vocal initiatives (a main vehicle of child involvement), and so explains why child language dominance in English may not have predicted child involvement. Mother-child physiological synchrony had significant predictive value for child responsiveness (not involvement), and even then it ranked low in the predictive value hierarchy. However, among children who showed English dominance, in the Englishlanguage play sessions physiological synchrony between mother and child may be interpreted to have preserved optimal child emotional availability when maternal emotional availability was not optimal. This pattern is seen in the tree model that predicted child responsiveness from child language dominance, maternal emotional availability, and mother-child physiological synchrony (Figure 1A). Among children dominant in English with mothers who did not optimally structure dyadic interactive play, lower dyadic physiological synchrony predicted greater child responsiveness whereas greater dyadic physiological synchrony predicted lesser child responsiveness. These results suggest the importance of physiological asynchrony in nonoptimal situations and, in fact, accord with those of Wilson et al. (2018) who showed that greater physiological synchrony between disagreeing partners in conflict (a non-optimal condition) predicted a heightened inflammatory profile and greater negative emotions. Therefore, physiological asynchrony between the child and mother in a non-optimal circumstance, that is, when maternal emotional availability is nonoptimal, appears to contribute to optimal child emotional availability. Overall, these results suggest that greater mother-child physiological synchrony might complement optimal motherchild emotional availability. However, it is also important to note that mother-child physiological synchrony had no predictive value for child emotional availability in the non-dominant language context. This finding highlights the fact that a deeper understanding of physiological processes underlying physiological synchrony is still wanting, and there is need for consensus to better understand and measure physiological synchrony (Azhari et al., 2019, 2020).

Before moving on to discuss predictions of maternal emotional availability, we note one additional salutary support of the Specificity Principle. In our design, the same mother-child dyads were seen in two play conditions, and two kinds of child emotional availability were coded from those play sessions. Comparisons of Figures $1 \mathrm{~A}$ with $1 \mathrm{C}$, and $1 \mathrm{~B}$ with $1 \mathrm{D}$, show that, within each play language session, child responsiveness and child involvement dimensions of emotional availability, respectively, were predicted by different patterns of the same set of predictors. As the Specificity Principle asserts, different developmental outcomes (qua dependent variables) can be predicted even by the same constellation of predictors (qua independent variables) under different setting conditions.

\section{Maternal Emotional Availability}

Next, we analyzed hierarchical relations among child language dominance, child emotional availability, and mother-child physiological synchrony in predicting overall maternal emotional availability. Here, as predicted by specificity as well, relations among the three independent variables differed in the two contexts (English-language and Mandarinlanguage play). 
In accord with our second hypothesis, during English-language play, which was the dominant language for majority of the children, child language dominance was more predictive of maternal emotional availability than child emotional availability. The less dominant the language, the lower the overall maternal emotional availability, and interactions using the dominant language of the child would elicit greater maternal emotional availability. This explanation (which needs to be empirically tested for confirmation) may be plausible because mothers tend to be more engaged with children who feel more comfortable in a task. Although second in priority for predicting, greater child responsiveness predicted greater maternal emotional availability, again lending support to what has been established in literature, viz. that child emotional availability is associated with maternal emotional availability (Engfer \& Gavranidou, 1987; Tamis-LeMonda et al., 2009). In the Mandarin-language play session, which was the non-dominant language for most children, child emotional availability (specifically child involvement) was more important in predicting maternal emotional availability than child language dominance. Furthermore, children who were less involved had mothers who were less emotionally available. Participants in a dyadic relationship influence each other in a bidirectional manner (Saunders et al., 2015). Biringen \& Robinson (1991) argued that a mother is not really highly sensitive unless her child is emotionally responsive. However, parental qualities as well as child qualities are certainly related to the specific interactional context and vary accordingly (Bornstein et al., 2007).

Before concluding, we point out that greater language dominance in children in Mandarin predicted greater child responsiveness when maternal sensitivity was at what we regard as a mediocre level (Figure 1B), and greater child language dominance in Mandarin also predicted greater child involvement when maternal emotional availability was not optimal (low maternal sensitivity but high maternal nonintrusiveness; Figure 1C). Neither dimension of child emotional availability was optimal when children showed lesser language dominance in Mandarin and maternal sensitivity was nonoptimal. Specific to the linguistic context, language dominance in the child appears to protect optimal child emotional availability (as in Mandarin-language interaction).

To our knowledge, this study is the first to provide evidence that the protective capacity of language dominance in bilingual children when maternal emotional availability is non-optimal. A plausible explanation for this observation could stem from the much-debated bilingual advantage in socioemotional skills (Bialystok et al., 2009; de Bot, 2017; Han, 2010; Lorette \& Dewaele, 2020). Bilinguals' presumed advantage in socioemotional skills could be viewed as an extension of their advantage in executive functioning, mainly in emotional self-regulation (Chen et al., 2014). It may be that the dominant language in bilingual children serves as a protective factor through emotional selfregulation. This speculative interpretation calls for in-depth research to understand exactly how language dominance spills over to enhance emotional self-regulation in bilingual children. Finally, we note that, although second in priority in predicting overall maternal emotional availability (Figure 2B), maternal emotional availability was higher when child language dominance in Mandarin was lower.

\section{Limitations}

Our findings are hemmed by some limitations (i.e., the small sample size, and children who were mostly dominant in English compared to Mandarin), and further research needs to confirm these predictive findings with larger samples as well as samples that are dominant in Mandarin compared to English and (perhaps) with samples that are balanced in English and Mandarin dominance. Additionally, knowing the mother's dominant language may be useful for future studies. Although it is assumed that parents tailor their language to their children's needs to maximize the efficacy of their verbal stimulation during parent-child interaction (e.g., de Falco et al., 2010; 2011; Topping et al., 2013), assessing mothers' language dominance may provide additional explanation of mother-child emotional variability during play in different language contexts. Despite these limitations, we believe that continuing this line of research can be worthwhile for learning more about specificities 
(and perhaps commonalities) in mother-child interaction in different linguistic contexts.

\section{Conclusions}

In support of Specificity Principle predictions, our results showed that patterns of hierarchical relations among child language dominance, emotional availability, and mother-child physiological synchrony predict the same dependent variables (mother or child emotional availability) in the same mother-child dyads differently in two contrasting contexts (English vs Mandarin language play). To our knowledge, this is the first study to give evidence of the protective capacity of language dominance in bilingual children when maternal emotional availability is non-optimal.

\section{References}

Altan, A., \& Hoff, E. (2018). Complex structures in the child-directed speech of native and nonnative speakers. In A. Altan \& E. Hoff (Eds.), Psycholinguistics and cognition in language processing (pp. 127-139). IGI Global.

Azhari, A., Truzzi, A., Neoh, J. M., Balagtas, J. P. M., Tan, H. A. H., Goh, P. L. P., Ang, X. H. A., Setoh, P., Rigo, P., Bornstein, M. H., \& Esposito, G. (2020). A decade of infant neuroimaging research: What have we learned and where are we going? Infant Behavior and Development, $\quad 58,101389$. https://doi.org/10.1016/j.infbeh.2019.101389

Azhari, A., Leck, W. Q., Gabrieli, G., Bizzego, A., Rigo, P., Setoh, P., Bornstein, M. H., \& Esposito, G. (2019). Parenting stress undermines mother-child brain-to-brain synchrony: A hyperscanning study. Scientific Reports, 9 , 11407. https://doi.org/10.1038/s41598-019-47810-4

Baker, C. (2014). A parents' and teachers' guide to bilingualism (4th ed.). Multilingual Matters.

Bialystok, E., Craik, F. I., Green, D. W., \& Gollan, T. H. (2009). Bilingual

minds. Psychological Science in the Public Interest, $\quad 10(3)$, 89-129. https://doi.org/10.1177/1529100610387084

Biringen, Z. (2000). Emotional availability: Conceptualization and research $\begin{array}{crr}\text { findings. } & \text { American } & \text { Journal of } \\ \text { Orthopsychiatry, } & 70(1), & 104-114 .\end{array}$ https://doi.org/10.1037/h0087711

Biringen, Z. (2008). Emotional availability scales (4th ed.). International Center for Excellence in Emotional Availability.

Biringen, Z., \& Robinson, J. (1991). Emotional availability in mother-child interactions: A reconceptualization for research. American journal of Orthopsychiatry, 61(2), 258271. https://doi.org/10.1037/h0079238

Bizzego, A., Gabrieli, G., Azhari, A., Setoh, P., \& Esposito, G. (2019). Computational methods for the assessment of empathic synchrony. In A. Esposito, M. Faundez- Zanuy, F. C. Morabito \& E. Pasero (Eds.), Progresses in Artificial Intelligence and Neural Systems (pp. 555-564). Springer.

Bosch, L., \& Sebastián-Gallés, N. (2001). Evidence of early language discrimination abilities in infants from bilingual environments. Infancy, 2(1), 29-49. https://doi.org/10.1207/S15327078IN0201_3

Bornstein, M. H. (2007). On the significance of social relationships in the development of children's earliest symbolic play: An ecological perspective. In A. Gönçü \& S. Gaskins (Eds.), Play and development: Evolutionary, sociocultural, and functional perspectives (pp. 101-129). Lawrence Erlbaum Associates. https://doi.org/10.4324/9780203936511

Bornstein, M. H. (2013a). Mother-infant attunement: A multilevel approach via body, brain, and behavior. In M. Legerstee, D. W. Haley, \& M. H. Bornstein (Eds.), The infant mind: Origins of the social brain (pp. 266-298). New York: Guilford.

Bornstein, M. H. (2013b). The specificity principle in multiple language learning. $S R C D$ Social Policy Report, 27(4), 5.

Bornstein, M. H. (2015). Children's parents. In M. H. Bornstein, T. Leventhal \& R. M. Lerner (Eds.), Handbook of child psychology and developmental science, Volume 4, Ecological settings and processes in developmental systems. (7th ed., pp. 55-132). Wiley. https://doi.org/10.1002/9781118963418.childpsy 403

Bornstein, M. H. (2017). The specificity principle in acculturation science. Perspectives on Psychological Science, 12(1), 3-45. https://doi.org/10.1177/1745691616655997 
Bornstein, M. H., Haynes, O. M., O’Reilly, A. W., \& Painter, K. (1996). Solitary and collaborative pretense play in early child-hood: Sources of individual variation in the development of representational competence. Child Development, 67, 2910-2929.

Bornstein, M.H., Hendricks, C., Haynes, O. M., \& Painter, K. M. (2007). Maternal sensitivity and child responsiveness: Associations with social context, maternal characteristics, and child characteristics in a multivariate analysis. Infancy, 12(2), 189-223. https://doi.org/10.1111/j.15327078.2007.tb00240.x

Bornstein, M. H., Putnick, D. L., Park, Y., Suwalsky, J. T., \& Haynes, O. M. (2017). Human infancy and parenting in global perspective: Specificity. Proceedings of the Royal Society B: Biological Sciences, 284(1869), 20172168. https://doi.org/10.1098/rspb.2017.2168

Bornstein, M. H., Suwalsky, J. T., \& Breakstone, D. A. (2012). Emotional relationships between mothers and infants: Knowns, unknowns, and unknown

unknowns. Development and psychopathology, 24(1), 113-123. https://doi.org/10.1017/S0954579411000708

Caldera, Y. M., Huston, A. C., \& O'Brien, M. (1989). Social interactions and play patterns of parents and toddlers with feminine, masculine, and neutral toys. Child Development, 60(1), 7076. https://doi.org/10.2307/1131072

Chen, S. H., Kennedy, M., \& Zhou, Q. (2012). Parents' Expression and discussion of emotion in the multilingual family: Does language matter? Perspectives on Psychological Science, 7(4), 365-383.

https://doi.org/10.1177/1745691612447307

Cheng, M., Setoh, P., Bornstein, M. H., \& Esposito, G. (2020). She thinks in english, but she wants in mandarin: Differences in Singaporean bilingual English-Mandarin maternal mental-state-talk. Behavioral Sciences, 10(7), 106. https://doi.org/10.3390/bs10070106

Creaven, A., Skowron, E. A., Hughes, B. M., Howard, S., \& Loken, E. (2014). Dyadic concordance in mother and preschooler resting cardiovascular function varies by risk status. Developmental Psychobiology, 56(1), 142-152. https://doi.org/10.1002/dev.21098

Cohen, J. (1960). A coefficient of agreement for nominal scales. Educational and
Psychological Measurement, 20(1), 37-46. https://doi.org/10.1177/001316446002000104

Cohen, J. (1968). Weighted kappa: Nominal scale agreement with provision for scaled disagreement or partial credit. Psychological Bulletin, 70(4), 213-220. https://doi.org/10.1037/h0026256

Costello T. J., Swartz M. D., Sabripour M., Gu X., Sharma R., \& Etzel C. J. (2003). Use of treebased models to identify subgroups and increase power to detect linkage to cardiovascular disease traits. BMC Genetics, 4(1), S66. https://doi.org/10.1186/1471-2156-4-S1-S66.

De Bot, K. (2017). Complexity Theory and Dynamic Systems Theory: Same or different? In L. Ortega \& Z. Han (Eds.), Complexity theory and language development: In celebration of Diane Larsen-Freeman (pp. 51-58). John Benjamins.

De Falco S., Esposito G., Venuti P., \& Bornstein M. H. (2010). Mothers and fathers at play with their children with Down syndrome: influence on child exploratory and symbolic activity. Journal of Applied Research in Intellectual Disabilities, 23(6), 597-605. https://doi.org/10.1111/j.1468-3148.2010.00558.x

De Falco, S., Venuti, P., Esposito, G., \& Bornstein, M. H. (2011). Maternal and paternal pragmatic speech directed to young children with Down syndrome and typical development. Infant Behavior and Development, 34(1), 161-169. https://doi.org/10.1016/j.infbeh.2010.12.002

De Houwer, A. (2015). Harmonious bilingual development: Young families' well-being in language contact situations. International Journal of Bilingualism, 19(2), 169-184. https://doi.org/10.1177/1367006913489202

De Houwer, A., \& Bornstein, M. H. (2016). Bilingual mothers' language choice in childdirected speech: Continuity and change. Journal of Multilingual and Multicultural Development, 37(7), 680-693. https://doi.org/10.1080/01434632.2015.1127929

Singapore. Department of Statistics Singapore. (2019). Key Household Income Trends, 2019. https://www.singstat.gov.sg//media/files/publications/households/pp-s26.pdf

Dewaele, J. (2008). The emotional weight of I love you in multilinguals' languages. Journal of Pragmatics, $\quad 40(10), \quad 1753-1780$. https://doi.org/10.1016/j.pragma.2008.03.002

Emde, R. N. (1980). Emotional availability: A reciprocal reward system for infants and parents 
with implications for prevention of psychosocial disorders. In. P.M. Taylor (Ed.), Parent-infant relationships (pp. 87-115). Grune \& Stratton.

Emde, R. N. (2000). Next steps in emotional availability research. Attachment \& Human Development, 2(2), 242-248. https://doi.org/10.1080/14616730050085590

Emde, R. N., \& Easterbrooks, M. A. (1985). Assessing Emotional Availability in Early Development. In R. N. Emde, W. K. Frankenburg \& J. Sullivan (Eds.), Early identification of children at risk: An international perspective (pp. 79-102). Springer.

Engfer, A., \& Gavranidou, M. (1987). Antecedents and consequences of maternal sensitivity: A longitudinal study. Advances in Psychology, $\quad 46, \quad 71-99$. https://doi.org/10.1016/s0166-4115(08)60346-5

Esposito, G., Manian, N., Truzzi, A., \& Bornstein, M. H. (2017). Response to infant cry in

clinically depressed and non-depressed mothers. PloS ONE, 12(1), e0169066. https://doi.org/10.1371/journal.pone.0169066

Feldman, R. (2007). Parent-infant synchrony and the construction of shared timing; physiological precursors, developmental outcomes, and risk conditions. Journal of Child Psychology and Psychiatry, 48(3-4), 329-354. https://doi.org/10.1111/j.1469-7610.2006.01701.x

Feldman, R., Singer, M., \& Zagoory, O. (2010). Touch attenuates infants' physiological reactivity to stress. Developmental Science, 13(2), 271-278. https://doi.org/10.1111/j.14677687.2009.00890.x

Fenson, L., Marchman, V. A., Thal, D. J., Dale, P. S., Reznick, J. S., \& Bates, E. (2007).

MacArthur-Bates Communicative Development Inventories: User's guide and technical manual (2nd ed.). Brookes.

Fuertes, M., Castro, S., Alves, M. J., Faria, A., Osório, T., \& Sousa, O. (2017). Interaction and language directed at children aged 15 months. Psicologia USP, 28(3), 346-357. https://doi.org/10.1590/0103-656420160154

Golland, Y., Keissar, K., \& Levit-Binnun, N. (2014). Studying the dynamics of autonomic activity during emotional experience. Psychophysiology, 51(11), 1101-1111. https://doi.org/10.1111/psyp.12261
Grüter, T., \& Paradis, J. (Eds.). (2014). Input and experience in bilingual development. John Benjamins.

Han, W. J. (2010). Bilingualism and socioemotional well-being. Children and Youth Services Review, 32(5), 720-731. https://doi.org/10.1016/j.childyouth.2010.01.009

Hua, Z. (2008). Duelling languages, duelling values: Code-switching in bilingual intergenerational conflict talk in diasporic families. Journal of Pragmatics, 40(10), 17991816.

https://doi.org/10.1016/j.pragma.2008.02.007

Leclère, C., Viaux, S., Avril, M., Achard, C., Chetouani, M., Missonnier, S., \& Cohen, D.

(2014). Why synchrony matters during mother-child interactions: A systematic review. PloS ONE, 9(12), e113571. https://doi.org/10.1371/journal.pone.0113571

Longhi, E. (2009). 'Songese': Maternal structuring of musical interaction with infants. Psychology of Music, 37(2), 195-213. https://doi.org/10.1177/0305735608097042

Lorette, P., \& Dewaele, J. M. (2020). Emotion recognition ability across different modalities: The role of language status (L1/LX), proficiency and cultural background. Applied Linguistics Review, 11(1), 1-26. https://doi.org/10.1515/applirev-2017-0015

Pavlenko, A. (2012). Affective processing in bilingual speakers: Disembodied cognition? International Journal of Psychology, 47(6), 405428.

https://doi.org/10.1080/00207594.2012.743665

Portes, A., \& Rumbaut, R. G. (2001). Legacies: The story of the immigrant second generation. University of California Press.

Sameroff, A. (Ed.). (2009). The transactional model of development: How children and contexts shape each other. American Psychological Association.

Sameroff, A. (2010). A unified theory of development: A dialectic integration of nature and nurture. Child Development, 81, 6-22. https://doi.org/10.1111/j.1467-8624.2009.01378.x

Saunders, H., Kraus, A., Barone, L., \& Biringen, Z. (2015). Emotional availability: Theory, research, and intervention. Frontiers in Psychology, $\quad 6, \quad 1069$. https://doi.org/10.3389/fpsyg.2015.01069

Setoh, P., Cheng, M., Bornstein, M., \& Esposito, G. (in press). Contrasting lexical biases 
in bilingual English-Mandarin speech: Verbbiased mothers, but noun-biased toddlers. Journal of Child Language.

Stack, D. M., Serbin, L. A., Girouard, N., Enns, L. N., Bentley, V. M. N., Ledingham, J. E., \& Schwartzman, A. E. (2012). The quality of the mother-child relationship in high-risk dyads: Application of the emotional availability scales in an intergenerational, longitudinal study. Development and Psychopathology, 24(1), 93105. https://doi.org/10.1017/s095457941100068x

Suveg, C., Shaffer, A., \& Davis, M. (2015). Family stress moderates relations between physiological and behavioral synchrony and child self-regulation in mother-preschooler dyads. Developmental Psychobiology, 58(1), 83-97. https://doi.org/10.1002/dev.21358

Tamis-LeMonda, C. S., Briggs, R. D., McClowry, S. G., \& Snow, D. L. (2009). Maternal control and sensitivity, child gender, and maternal education in relation to children's behavioral outcomes in African American families. Journal of Applied Developmental Psychology, 30(3), 321-331. https://doi.org/10.1016/j.appdev.2008.12.018

Tardif, T., \& Fletcher, P. (2008) Chinese communicative development inventories: User's guide and manual. Peking University Medical Press.

Teng, A. (2016, July 10). Singapore kids using more English but strong in Chinese: Study. The Straits Times. https://www.straitstimes.com/singapore/educatio $\mathrm{n} /$ spore-kids-using-more-english-but-strong-inchinese

Thompson, L. A., \& Trevathan, W. R. (2009). Cortisol reactivity, maternal sensitivity, and infant preference for mother's familiar face and rhyme in 6-month-old infants. Journal of Reproductive and Infant Psychology, 27( 2), 143-167. https://doi.org/10.1080/02646830801918463

Tokuhama-Espinosa, T. (Ed.). (2003). The multilingual mind: issues discussed by, for, and about people living with many languages. Greenwood Publishing Group.

Topping, K., Dekhinet, R., \& Zeedyk, S. (2013). Parent-infant interaction and children's language development. Educational Psychology, 33(4), 391-426. https://doi.org/10.1080/01443410.2012.744159 Venuti, P., De Falco, S., Esposito, G., \& Bornstein, M. H. (2009). Mother-child play:
Children with Down syndrome and typical development. American Journal on Intellectual and Developmental Disabilities, 114(4), 274-288. https://doi.org/10.1352/1944-7558-114.4:274-288

Wilson, S. J., Bailey, B. E., Jaremka, L. M., Fagundes, C. P., Andridge, R., Malarkey, W. B., \& Kiecolt-Glaser, J. K. (2018). When couples' hearts beat together: Synchrony in heart rate variability during conflict predicts heightened inflammation throughout the day. Psychoneuroendocrinology, 93, 107-116. https://doi.org/10.1016/j.psyneuen.2018.04.017

Woltering, S., Lishak, V., Elliott, B., Ferraro, L., \& Granic, I. (2015). Dyadic attunement and physiological synchrony during mother-child interactions: An exploratory study in children with and without externalizing behavior problems. Journal of Psychopathology and Behavioral Assessment, $\quad 37$, 624-633. https://doi.org/10.1007/s10862-015-9480-3 
Figure 1

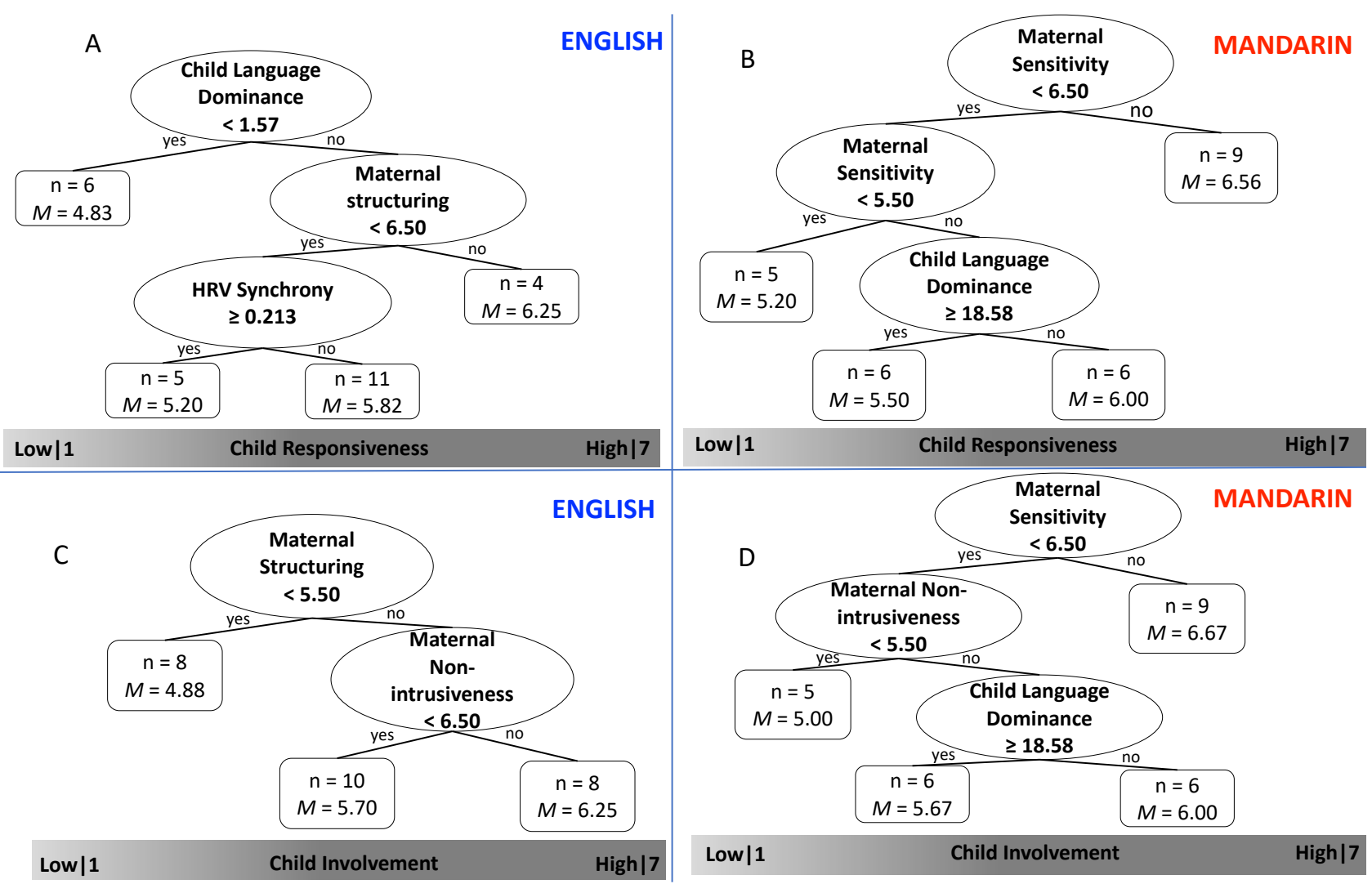

Figure 1. The optimal tree that predicts child emotional availability (Responsiveness and Involvement) during English or Mandarin language play sessions. (A) Model for child responsiveness during the English-language play session. (B) Model for child responsiveness during the Mandarin-language play session. (C) Model for child involvement during the English-language play session. (D) Model for child involvement during the Mandarin-language play session. The bottom rectangle shows the distribution of the child emotional availability dimension from lower (left) to higher (right). The values in oval leaves of the tree refer to the condition of the independent variables (child language dominance, maternal emotional availability, and mother-child physiological synchrony) that statistically divides the distribution of the dependent variable (dimension of child emotional availability). Below each oval leaf, the indications "yes" or "no" refer to whether or not the condition is met. Each leaf is divided in two sub-leaves. The terminal leaves (quadrangles) represent subgroups that cannot be further subdivided. The $\mathrm{N}$ value in the terminal leaves represents the size of the group, and $\mathrm{M}$ is the mean value of the group for the dependent variable. 
Figure 2

A

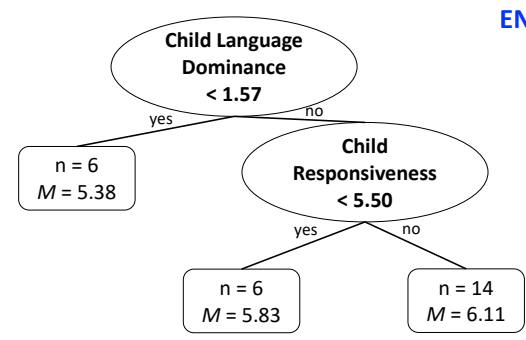

Low|1
ENGLISH

High|7
B

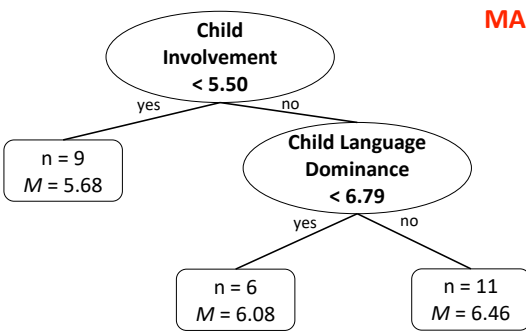

Overall Maternal Emotional Availability
MANDARIN

Figure 2.The optimal tree that predicts mother emotional availability (overall score) during English or Mandarin language play sessions. (A) Model for overall maternal emotional availability during the English-language play session. (B) Model for overall maternal emotional availability during the Mandarin-language play session. The bottom rectangle shows the distribution of maternal emotional availability from lower (left) to higher (right). The values in oval leaves of the tree refer to the condition of the independent variables (child language dominance, child emotional availability, and mother-child physiological synchrony) that statistically divides the distribution of the dependent variable (overall maternal emotional availability). Below each oval leaf, the indications "yes" or "no" refer to whether or not the condition is met. Each leaf is divided in two sub-leaves. The terminal leaves (quadrangles) represent subgroups that cannot be further subdivided. The $\mathrm{N}$ value in the terminal leaves represents the size of the group, and $\mathrm{M}$ is the mean value of the group for the dependent variable. 